\title{
Childhood disability and socio-economic circumstances in low and middle income countries: systematic review
}

\author{
Douglas E Simkiss ${ }^{1 *}$, Clare M Blackburn², Felix O Mukoro ${ }^{3}$, Janet M Read ${ }^{4}$ and Nicholas J Spencer ${ }^{5}$
}

\begin{abstract}
Background: The majority of children with disability live in low and middle income (LAMI) countries. Although a number of important reviews of childhood disability in LAMI countries have been published, these have not, to our knowledge, addressed the association between childhood disability and the home socio-economic circumstances (SEC). The objective of this study is to establish the current state of knowledge on the SECs of children with disability and their households in LAMI countries through a systematic review and quality assessment of existing research.

Methods: Electronic databases (MEDLINE; EMBASE; PUBMED; Web of Knowledge; Psyclnfo; ASSIA; Virtual Health Library; POPLINE; Google scholar) were searched using terms specific to childhood disability and SECs in LAMI countries. Publications from organisations including the World Bank, UNICEF, International Monetary Fund were searched for. Primary studies and reviews from 1990 onwards were included. Studies were assessed for inclusion, categorisation and quality by 2 researchers.

Results: 24 primary studies and 13 reviews were identified. Evidence from the available literature on the association between childhood disability and SECs was inconsistent and inconclusive. Potential mechanisms by which poverty and low household SEC may be both a cause and consequence of disability are outlined in the reviews and the qualitative studies. The association of poor SECs with learning disability and behaviour problems was the most consistent finding and these studies had low/medium risk of bias. Where overall disability was the outcome of interest, findings were divergent and many studies had a high/medium risk of bias. Qualitative studies were methodologically weak.

Conclusions: This review indicates that, despite socially and biologically plausible mechanisms underlying the association of low household SEC with childhood disability in LAMI countries, the empirical evidence from quantitative studies is inconsistent and contradictory. There is evidence for a bidirectional association of low household SEC and disability and longitudinal data is needed to clarify the nature of this association.
\end{abstract}

\section{Background}

In 2004 the Global Burden of Disease report estimated that over 100 million children under the age of 15 years had a moderate or severe disability, the majority of whom live in low and middle income countries (LAMI) $[1,2]$. Research on these children in LAMI countries however, has been described as 'woefully inadequate' [3]. Reviews of research have found that the majority of

\footnotetext{
* Correspondence: d.e.simkiss@warwick.ac.uk

${ }^{1}$ Health Sciences Research Institute, Warwick Medical School, University of

Warwick, (Gibbet Hill Road), Coventry, (CV4 7AL), UK

Full list of author information is available at the end of the article
}

studies have focused on cross-sectional, communitybased epidemiologic studies aimed at finding the prevalence or aetiology of certain conditions or impairments [3]. In addition, the quality of many studies has been judged inadequate [3-5]. It has been argued that it is important to move beyond prevalence studies to generate informative data on the circumstances of people with disability, including how these compare with those of their non-disabled peers [5]. There is now widespread acceptance that disability definitions and measures should no longer focus solely on impairments and other individual characteristics and conditions, important
C Biomed Central 
though those undoubtedly are. Definitions and measures of disability should also incorporate contextual dimensions which may enable or act as barriers to disabled children's participation and human rights [6-9]. A paradigm of disability, which incorporates both individual characteristics and social circumstances, is reflected in the recently developed International Classification of Functioning Disability and Health - Children and Youth (ICF-CY) [10] and the United Nations Convention on the Rights of Persons with Disabilities (UNCRPD) [11]. These factors make it an imperative to remedy the significant knowledge gap about the lives of children with disability in LAMI countries [1,2,12]. The World Disability Report [1] places emphasis on the role of environmental factors in disability; however, it identifies the absence of clarity on the relationship between household socio-economic circumstances and disability among both children and adults in developing countries. An important starting point is to scope existing studies that address this relationship.

\section{Objective}

A number of important reviews of childhood disability in LAMI countries have already been undertaken and point to significant gaps in knowledge about this important group of children and their families [3,5,13-15]. The purpose of this paper is to establish the current state of knowledge on the socio-economic circumstances (SEC) of disabled children and their households in LAMI countries through a systematic review and quality assessment of existing research. We are taking a broad definition of disability consistent current thinking in the World Report on Disability and for the definition of SEC, we include the following variables; income/asset measures, parental education, poverty, area-based measures of socio-economic factors, occupation-based measures and housing-based measures. To our knowledge, this is the first review specifically reporting the association between childhood disability and the home socioeconomic circumstances in LAMI countries.

\section{Methods}

We carried out a review of childhood disability in LAMI countries focusing on four areas: methodological issues in studying childhood disability; household SEC of children with disabilities; the rights of children with disabilities; the use of the International Classification of Functioning, Disability and Health - Children and Young people (ICF-CY) in relation in LAMI countries. This paper is concerned only with the household SEC of children with disability in LAMI countries. Further papers will address the literature related to the rights of children with disability and the use of the ICF-CY. The review by Maulik and Darmstadt [3] covers the methodological issues in detail and our review does not add significantly to their conclusions.

\section{Search Strategy \\ Databases searched}

The search for evidence explored six electronic databases, including medical literature (MEDLINE, EMBASE, and PUBMED), Web of Knowledge and CSA databases (PsycInfo, ASSIA). Broad searches were also conducted in Virtual Health Library, and POPLINE databases, as well as in Google scholar http://scholar. google.co.uk. Publications from organisations such as World Health Organisation (WHO), The World Bank and International Monetary Fund (IMF), United Nations Children's Fund (UNICEF); UN Statistics Division; Statistical Information and Monitoring Programme on Child Labour (SIMPOC); Development Banks (InterAmerican; African; Asian); Save the Children; Washington Group; Paris21 consortium (Partnership in Statistics for Development in the 21st. century) and The Foundation for Scientific and Industrial Research (SINTEF) were screened. Bibliographies of selected articles were also searched. We set out to identify all relevant publications regardless of language. Only primary studies and reviews from LAMI countries evaluating SEC of disabled children, methodological issues in childhood disability data collection, the use of ICF-CY, and the rights of children with disability were considered eligible for review. Only literature published between 1990 and June 2009 were included because earlier papers may not now be relevant and the United Nations Convention on the Rights of the Child, the basis of children's rights, was published in 1989.

\section{Search terms used}

The search terms and strategies used for the searched databases are summarized in Table 1.

\section{Inclusion criteria}

Both primary studies and reviews relating to household SEC of children with disability in LAMI countries were included. Only quantitative studies with data on the association of childhood disabling conditions and household SEC were included. Seven studies that reported on children and adults combined with no separate analysis of childhood disability and household SEC were excluded. Qualitative studies and reviews were included if they reported on childhood and adult disability and household SEC.

\section{Instrumentation}

The studies were independently assessed for inclusion and categorization by two researchers, DS and NS. There was a very high level of $96 \%$ agreement overall and $92 \%$ for included studies, final decisions were made by agreement. All included studies were entered into a spreadsheet and then categorised using variables that included details of the research design, screening tools/ 
Table 1 Search terms by databases searched

\begin{tabular}{|c|c|}
\hline Database & Search strategy \\
\hline MEDLINE & $\begin{array}{l}\text { exp Disabled Children/or exp Disabled Persons/ } \\
\text { exp Sensation Disorders/or exp Learning Disorders/ } \\
\text { exp Physical Impairment/ } \\
\text { ICF-CY.mp. or international classification of function.mp. } \\
\text { exp Disability Evaluation/ } \\
\text { child rights.mp. or exp Human Rights/ } \\
\text { disability rights.mp. } \\
1 \text { or } 2 \text { or } 3 \text { or } 4 \text { or } 5 \text { or } 6 \text { or } 7 \\
\text { exp Developing Countries/ } \\
8 \text { AND } 9 \\
\text { limit } 10 \text { to (yr="1990 - 2009" and ("infant (1 to } 23 \text { months)" or "preschool child (2 to } 5 \text { years)" or "child (6 to } 12 \text { years)" or } \\
\text { "adolescent }(13 \text { to } 18 \text { years)")) }\end{array}$ \\
\hline EMBASE & $\begin{array}{l}\text { exp Handicapped Child/ } \\
\text { exp Sensory Dysfunction/ } \\
\text { exp Intellectual Impairment/or exp Hearing Impairment/ } \\
\text { exp Disability/or exp Physical Disability/or exp Mental Deficiency/ } \\
\text { exp Functional Assessment/or exp "International Classification of Functioning, Disability and Health"/or exp Classification/or } \\
\text { ICF-CY.mp. } \\
\text { exp Human Rights/or child rights.mp. } \\
\text { disability rights.mp. } \\
6 \text { or } 7 \text { or } 4 \text { or } 1 \text { or } 5 \text { or } 3 \text { or } 2 \\
\text { exp Developing Country/or low-income countries.mp. } \\
9 \text { and } 8 \\
\text { limit } 10 \text { to (yr="1990 - } 2009^{\prime \prime} \text { and (infant < to one year }>\text { or preschool child }<1 \text { to } 6 \text { years }>\text { or school child }<7 \text { to } 12 \text { years } \\
>\text { or adolescent }<13 \text { to } 17 \text { years }>\text { ) }\end{array}$ \\
\hline PUBMED & $\begin{array}{l}\text { ("developing countries"[MeSH Major Topic]) AND (("sensation disorders"[MeSH Major Topic]) OR ("mental retardation"[MeSH } \\
\text { Major Topic])) OR ("disabled children"[MeSH Major Topic])) } \\
\text { Limits: Infant: 1-23 months, Preschool Child: 2-5 years, Child: 6-12 years, Adolescent: 13-18 years }\end{array}$ \\
\hline $\begin{array}{l}\text { VHL/POPLINE/Google } \\
\text { Scholar }\end{array}$ & ("disabled children" OR "disability" OR "ICF-CY") AND ("developing countries") \\
\hline CSA (Psyclnfo, ASSIA) & 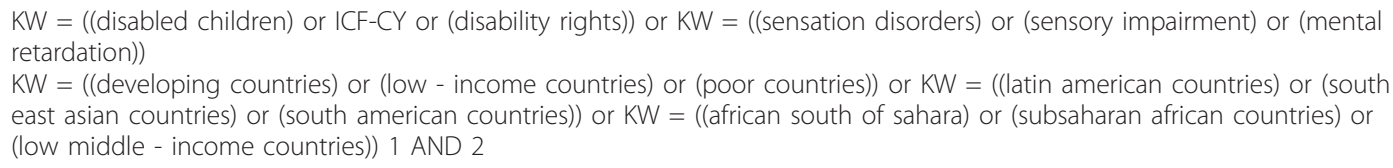 \\
\hline
\end{tabular}

method used, primary population studied, specific health condition and broad aim. The key focuses of the papers were categorized according to the broad themes including methodology, household socio-economic circumstances, rights, and ICF/ICF-CY. This paper is concerned only with those papers focusing on the household socioeconomic circumstances of children with disabilities.

\section{Quality assessment}

Papers were assessed for quality using the STROBE criteria http://www.strobe-statement.org modified for the specific requirements of the review (Table 2). Studies were judged to have low risk of bias if they had optimum quality in at least 6 out of the eight quality domains and no domains with least valuable quality. Studies with medium risk of bias were those with less than six optimum quality domains with only one least valuable quality domain or six optimum but with one least valuable domain. High risk of bias was defined as more than one least valuable domain or case series with no controls or comparison group.

\section{Analysis}

Summary findings related to the household SEC of disabled children in developing countries were extracted from primary studies and reviews identified by the search. The findings of qualitative studies were summarised. The association of household SEC with childhood disability was expressed as odds ratios with 95\% confidence intervals where available or $\mathrm{p}$ values where odds ratios were not stated by the authors. Meta-analysis of the findings of the empirical studies was not attempted due to heterogeneity of the studies.

\section{Results}

24 primary studies of the relationship of childhood disability with family and household social circumstances in LAMI countries were identified [2,4,16-38] (see Table 3 ). We found no reviews focusing specifically on the relationship of childhood disability with family and household social circumstances in LAMI countries. Three reviews [39-41] set out to address the association of both adult and child disability with poverty in LAMI countries; a further 10 reviews [3,5,13-15,42-46] refer to 
Table 2 Quality assessment criteria

\begin{tabular}{|c|c|c|c|}
\hline Quality criterion & Optimum & Adequate & Least valuable \\
\hline $\begin{array}{l}\text { Design \& data } \\
\text { collection methods }\end{array}$ & Longitudinal & $\begin{array}{l}\text { Cross-sectional survey or case-control } \\
\text { study }\end{array}$ & $\begin{array}{l}\text { Case series without controls or } \\
\text { comparison groups }\end{array}$ \\
\hline Purpose & $\begin{array}{l}\text { Specifically designed to collect childhood } \\
\text { disability data }\end{array}$ & $\begin{array}{l}\text { General purpose survey including } \\
\text { questions on childhood disability }\end{array}$ & $\begin{array}{l}\text { Inadequate childhood disability } \\
\text { data }\end{array}$ \\
\hline $\begin{array}{l}\text { Data collection } \\
\text { methods }\end{array}$ & Interview based & Interview based & Postal questionnaire based \\
\hline $\begin{array}{l}\text { Quantitative/ } \\
\text { qualitative }\end{array}$ & $\begin{array}{l}\text { Combined quantitative \& qualitative data on } \\
\text { childhood disability }\end{array}$ & $\begin{array}{l}\text { Quantitative only for prevalence } \\
\text { estimation; qualitative only to study } \\
\text { impact of disability on household }\end{array}$ & $\begin{array}{l}\text { Either quantitative or qualitative } \\
\text { data that cannot be adequately } \\
\text { used to estimate prevalence or } \\
\text { impact }\end{array}$ \\
\hline $\begin{array}{l}\text { Definitions \& } \\
\text { classifications of } \\
\text { disability/ } \\
\text { impairment } \\
\end{array}$ & $\begin{array}{l}\text { Definition that is widely used, internationally } \\
\text { validated \& comparable with other data sets }\end{array}$ & $\begin{array}{l}\text { Less than optimal definition but } \\
\text { sufficiently detailed to allow reasonable } \\
\text { prevalence estimates to be made }\end{array}$ & $\begin{array}{l}\text { Unclear definition that does not } \\
\text { allow comparison with other data } \\
\text { sets }\end{array}$ \\
\hline Sampling \& sample & $\begin{array}{l}\text { Representative of households with children } \\
\text { in study country \& sufficient size to enable } \\
\text { prevalence estimates of less common } \\
\text { impairments. }\end{array}$ & $\begin{array}{l}\text { Representative of households with } \\
\text { children in study country }\end{array}$ & $\begin{array}{l}\text { Non-representative samples } \mathrm{OR} \\
\text { Small samples with inadequate } \\
\text { numbers to make reliable } \\
\text { prevalence estimates }\end{array}$ \\
\hline Response rates & $\begin{array}{l}\text { Full information on response rates with low } \\
\text { non-response }(<10 \%)\end{array}$ & $\begin{array}{l}\text { Limited information on response rates or } \\
\text { non-response }(10-20 \%)\end{array}$ & $\begin{array}{l}\text { No response information or high } \\
\text { response rates }(>20 \%)\end{array}$ \\
\hline $\begin{array}{l}\text { Household } \\
\text { measures of SES \& } \\
\text { socio-demographic } \\
\text { characteristics }\end{array}$ & $\begin{array}{l}\text { Data covering measures of income, parental } \\
\text { education, household wealth \& assets in } \\
\text { addition to other socio-demographic } \\
\text { characteristics including ethnicity, marital } \\
\text { status, parental age, parental disability }\end{array}$ & $\begin{array}{l}\text { Limited measures of SES such as maternal } \\
\text { education only OR SES measures but } \\
\text { limited data on other socio-demographic } \\
\text { characteristics }\end{array}$ & $\begin{array}{l}\text { No valid measures of SES or S-D } \\
\text { characteristics }\end{array}$ \\
\hline $\begin{array}{l}\text { Information on } \\
\text { child }\end{array}$ & Data on age, sex, school attendance & $\begin{array}{l}\text { Limited information on age but no other } \\
\text { data }\end{array}$ & $\begin{array}{l}\text { No data on child - only on } \\
\text { household as a whole }\end{array}$ \\
\hline $\begin{array}{l}\text { Information on } \\
\text { children }(<19 \\
\text { years) living } \\
\text { outside the home }\end{array}$ & Complete data on all children & Limited data on all children & $\begin{array}{l}\text { No data on children living outside } \\
\text { the home }\end{array}$ \\
\hline
\end{tabular}

the association within the context of reviewing other aspects of disability in LAMI countries.

Of the identified primary studies, 5 reported qualitative data within country level reports and 19 were quantitative studies conducted in more than 50 LAMI countries (Table 3). Five studies reported multi-country data, one of which [33] included developed countries as well as developing countries. The age range of children included in the quantitative studies varied from 0-20 years; eight studies reported on children under the age of 10 years, 2 on adolescents only and the remaining studies included young children as well as adolescents. The qualitative data included both adults and children; however, the ages of the children were not specified.

Two of the quantitative studies were based on prospective cohort studies [16,17] and one (reported in two papers) on a randomised control trial [27,28]; the remaining 16 were cross-sectional surveys or case-control studies (Table 3). A range of disabilities were studied in the quantitative studies; total disability rates were reported in 8 studies, emotional \& behavioural disorders and mental retardation in 6 , hearing loss in 2 , visual impairment in 1 , and neurological, neurodevelopment and musculoskeletal impairment in 4. The most commonly used instrument to measure disability in the quantitative surveys was the Ten Questions Questionnaire (TQQ); this was used in 8 studies, four as a screening tool in an initial phase of the study and four as the main measure of disability. The qualitative data were based on interviews and focus group including adults and children (or their parents) with a range of disabilities including hearing, visual, intellectual and motor impairment.

Eleven quantitative studies used multiple measures of household SEC. Parental education (mostly maternal education) and income/asset-based measures were the most commonly used measures. Six studies used areabased measures, two occupation-based measures and two housing-related measures. The poverty measures used in the reports containing qualitative data were not specified.

Although the reviews that primarily address disability and poverty [39-41] categorically state that poverty is both a cause and consequence of disability, they review very limited empirical data on the relationship and none related specifically to the household SEC of children 
Table 3 Primary studies of household SEC of children with disabilities

\begin{tabular}{|c|c|c|c|c|c|}
\hline Author/year/country & Study design & Population \& sample & Disability measure & $\begin{array}{l}\text { Measure of } \\
\text { household SEC }\end{array}$ & $\begin{array}{l}\text { Summary of results } \\
\text { [Odds ratios (OR) } \\
\text { with } 95 \% \text { Cls where } \\
\text { available] }\end{array}$ \\
\hline $\begin{array}{l}\text { Anselmi et al, } 2008 \\
\text { Brazil }^{16}\end{array}$ & $\begin{array}{l}\text { Prospective cohort } \\
\text { study }\end{array}$ & $\begin{array}{l}601 \text { children of } 634 \\
\text { randomly selected } \\
\text { from the Pelotas birth } \\
\text { cohort \& followed up } \\
\text { between the ages of } 4 \\
\& 12 \text { years. }\end{array}$ & $\begin{array}{l}\text { Emotional and } \\
\text { behavioural problems } \\
\text { measured using the } \\
\text { Child Behavior } \\
\text { Checklist (CBCL) } \\
\text { administered at } 4 \& 12 \\
\text { years of age }\end{array}$ & Family income & $\begin{array}{l}\text { Externalising (OR .72 } \\
(.52,96)) \text { and } \\
\text { internalising (OR . } 68 \\
(.47,98)) \text { behaviours } \\
\text { and attention } \\
\text { problems (OR } .57 \\
(.39,84)) \text { at age } 12 \\
\text { years were significantly } \\
\text { associated with low } \\
\text { family income at } 4 \\
\text { years }\end{array}$ \\
\hline $\begin{array}{l}\text { Bashir et al, } 2002 \\
\text { Pakistan }^{17}\end{array}$ & $\begin{array}{l}\text { Prospective cohort } \\
\text { study }\end{array}$ & $\begin{array}{l}772 \text { children aged 4-6 } \\
\text { years of age out of } \\
1476 \text { births enrolled in } \\
\text { a birth cohort in } 4 \\
\text { areas of Lahore with } \\
\text { contrasting socio- } \\
\text { economic } \\
\text { characteristics }\end{array}$ & $\begin{array}{l}\text { Mild Mental } \\
\text { Retardation (MMR) } \\
\text { measured as IQ in the } \\
\text { range 50-69 measured } \\
\text { using WISC \& Griffiths } \\
\text { tests among those } \\
\text { initially identified using } \\
\text { the Ten Question } \\
\text { screening test }\end{array}$ & $\begin{array}{l}\text { Four socio- } \\
\text { economically distinct } \\
\text { areas - village, } \\
\text { periurban slum, urban } \\
\text { slum \& upper middle } \\
\text { class area. [no } \\
\text { individual or } \\
\text { household level SES } \\
\text { data reported] }\end{array}$ & $\begin{array}{l}\text { MMR prevalence: } \\
\text { Upper middle class } \\
1.4 \% \\
\text { Village } 4.8 \% \\
\text { Urban slum } 6.1 \% \\
\text { Periurban slum } 10.5 \%\end{array}$ \\
\hline $\begin{array}{l}\text { Bastos et al, } 1995 \\
\text { Tanzania }^{18}\end{array}$ & Cross-sectional survey & $\begin{array}{l}854 \text { children aged } 6 \text { to } \\
16 \text { years in schools in } \\
\text { the Moshi and } \\
\text { Munduli districts of } \\
\text { northern Tanzania }\end{array}$ & $\begin{array}{l}\text { Hearing loss measured } \\
\text { by pneumotoscopy } \\
\text { and screening } \\
\text { audiometry }\end{array}$ & Urban v. Rural areas & $\begin{array}{l}\text { Hearing loss in speech } \\
\text { frequency range more } \\
\text { common in urban } \\
(37 \%) \text { compared with } \\
\text { rural }(18 \%) \text { and high } \\
\text { frequency loss also } \\
\text { more common in } \\
\text { urban compared with } \\
\text { rural }\end{array}$ \\
\hline $\begin{array}{l}\text { Durkin et al, } 1998 \\
\text { Pakistan }^{19}\end{array}$ & $\begin{array}{l}\text { Cross-sectional } \\
\text { population survey }\end{array}$ & $\begin{array}{l}\text { 6,365 2-9 year old } \\
\text { children in Greater } \\
\text { Karachi screened in } \\
\text { phase } 1 \text { of the survey } \\
\text { using TQQ; } 818 \\
\text { screening positive and } \\
545 \text { of those screening } \\
\text { negative assessed in } \\
\text { phase } 2\end{array}$ & $\begin{array}{l}\text { Identification of mental } \\
\text { retardation by: Phase 1: } \\
\text { TQQ screen Phase 2: } \\
\text { clinical assessment } \\
\text { using Stanford-Binet IQ } \\
\text { test \& adaptive } \\
\text { behaviour scale } \\
\text { developed for Pakistani } \\
\text { children }\end{array}$ & $\begin{array}{l}\text { Maternal education } \\
\text { level (Some v. None) } \\
\text { Urban v. Rural }\end{array}$ & $\begin{array}{l}\text { Mild Retardation (IQ } \\
\text { 50-70): No education } \\
\text { OR 3.08(1.85,6.14) Rural } \\
\text { OR 2.33(1.33,2.75) } \\
\text { Serious Retardation (IQ } \\
\text { < 50): No education } \\
\text { OR 3.25(1.86,8.43) Rural } \\
\text { OR 2.21(0.87,5.57) }\end{array}$ \\
\hline $\begin{array}{l}\text { Filmer } 2005 \\
9 \text { low \& middle } \\
\text { income countries } \\
\text { (Jamaica, Romania, } \\
\text { Cambodia, Indonesia, } \\
\text { Mozambique, } \\
\text { Burundi, Myanmar, } \\
\text { Mongolia and Sierra } \\
\text { Leone) }^{20}\end{array}$ & $\begin{array}{l}11 \text { Nationally } \\
\text { representative cross- } \\
\text { sectional household } \\
\text { surveys in } 9 \text { countries - } \\
3 \text { Living Standards } \\
\text { Measurement Studies; } \\
3 \text { national socio- } \\
\text { economic status } \\
\text { surveys; } 4 \text { MICS } 2 \\
\text { surveys; } 1 \text { DHS survey }\end{array}$ & $\begin{array}{l}\text { Children \& young } \\
\text { people aged 6-17 } \\
\text { years - population } \\
\text { samples ranged from } \\
1,649 \text { in Jamaica (2000) } \\
\text { to } 64,136 \text { in Indonesia } \\
(2000)\end{array}$ & $\begin{array}{l}\text { Impairment definitions } \\
\text { of disability consistent } \\
\text { with ICF's 'body } \\
\text { functioning \& structure' } \\
\text { domain - range of } \\
\text { different questions } \\
\text { used }\end{array}$ & $\begin{array}{l}\text { Quintiles of Household } \\
\text { per capita } \\
\text { consumption } \\
\text { expenditure in LSMS \& } \\
\text { SES surveys } \\
\text { Quintiles of index of } \\
\text { household consumer } \\
\text { assets \& housing } \\
\text { characteristics in DHS } \\
\text { \& MICS2 }\end{array}$ & $\begin{array}{l}\text { Prevalence higher in } \\
\text { poorest quintile } \\
\text { compared with richest } \\
\text { in all countries except } \\
\text { Burundi, Cambodia, } \\
\text { Mongolia and } \\
\text { Mozambique - only } \\
\text { Indonesia shows a } \\
\text { clear social gradient } \\
\text { across quintiles }\end{array}$ \\
\hline $\begin{array}{l}\text { Filmer } 2008 \\
13 \text { low \& middle } \\
\text { income countries } \\
\text { (Jamaica, Romania, } \\
\text { Cambodia, Indonesia, } \\
\text { Mozambique, } \\
\text { Burundi, Mongolia, } \\
\text { South Africa, Chad, } \\
\text { India, Colombia, } \\
\text { Bolivia and Zambia) } \\
\text { [NB: some overlap }^{4} \\
\text { with Filmer 2005] }\end{array}$ & $\begin{array}{l}14 \text { Nationally } \\
\text { representative cross- } \\
\text { sectional household } \\
\text { surveys in } 13 \text { countries } \\
\text { - } 2 \text { Living Standards } \\
\text { Measurement Studies; } \\
5 \text { national socio- } \\
\text { economic status } \\
\text { surveys; } 2 \text { MICS } 2 \\
\text { surveys; } 5 \text { DHS surveys }\end{array}$ & $\begin{array}{l}\text { Children \& young } \\
\text { people aged 6-17 } \\
\text { years - population } \\
\text { samples ranged from } \\
5,865 \text { in Burundi (2000) } \\
\text { to } 140,297 \text { in India } \\
\text { (1992) }\end{array}$ & $\begin{array}{l}\text { Impairment definitions } \\
\text { of disability consistent } \\
\text { with ICF's 'body } \\
\text { functioning \& structure' } \\
\text { domain - range of } \\
\text { different questions } \\
\text { used }\end{array}$ & $\begin{array}{l}\text { Quintiles of Household } \\
\text { per capita } \\
\text { consumption } \\
\text { expenditure in LSMS \& } \\
\text { SES surveys } \\
\text { Quintiles of index of } \\
\text { household consumer } \\
\text { assets \& housing } \\
\text { characteristics in DHS } \\
\text { \& MICS2 }\end{array}$ & $\begin{array}{l}\text { Only Indonesia \& India } \\
\text { show clear differences } \\
\text { in disability prevalence } \\
\text { between poorest \& } \\
\text { richest quintiles - } \\
\text { otherwise non- } \\
\text { significant differences }\end{array}$ \\
\hline
\end{tabular}


Table 3 Primary studies of household SEC of children with disabilities (Continued)

\begin{tabular}{|c|c|c|c|c|c|}
\hline $\begin{array}{l}\text { Grut \& Ingstad, } 2006 \\
\text { Yemen }^{21}\end{array}$ & Qualitative study & $\begin{array}{l}28 \text { interviews involving } \\
38 \text { individuals in } \\
\text { households with } \\
\text { disabled people, \& one } \\
\text { group interview in an } \\
\text { institution for disabled } \\
\text { girls [15 children; } 20 \\
\text { adults; } 2 \text { Disabled } \\
\text { People's Organisations] }\end{array}$ & $\begin{array}{l}\text { Range of disabilities } \\
\text { including physical, } \\
\text { intellectual, hearing \& } \\
\text { visual } \\
\text { [interviews of those } \\
\text { with intellectual } \\
\text { impairments } \\
\text { conducted with } \\
\text { parents in presence of } \\
\text { the disabled person] }\end{array}$ & $\begin{array}{l}\text { Specific measures not } \\
\text { used but study does } \\
\text { examine the impact of } \\
\text { disability on the lives } \\
\text { of households with } \\
\text { disabled members } \\
\text { particularly children } \\
\text { and explores the } \\
\text { association of poverty } \\
\text { \& disability }\end{array}$ & $\begin{array}{l}\text { Complex relationship } \\
\text { between disability and } \\
\text { poverty demonstrated } \\
\text { with insights into how } \\
\text { disability and poverty } \\
\text { interact to limit life } \\
\text { chances }\end{array}$ \\
\hline $\begin{array}{l}\text { Hackett et al, } 1999 \\
\text { India }^{22}\end{array}$ & $\begin{array}{l}\text { Random cluster } \\
\text { sampling for cross- } \\
\text { sectional survey }\end{array}$ & $\begin{array}{l}1326 \text { children aged 8- } \\
12 \text { years in } 2 \text { local } \\
\text { government districts } \\
\text { outside the city of } \\
\text { Calicut, Kerala State, } \\
\text { India }\end{array}$ & $\begin{array}{l}\text { Child psychiatric } \\
\text { disorder identified in } 2 \\
\text { phases: } \\
\text { Phase 1: Screening } \\
\text { interviews using } \\
\text { various instruments } \\
\text { including Rutter's A2 } \\
\text { scale plus Rutter } \\
\text { teacher completed B2 } \\
\text { scale } \\
\text { Phase 2: detailed } \\
\text { psychiatric assessment } \\
\text { of screen positive \& } 93 \\
\text { screen negative }\end{array}$ & $\begin{array}{l}\text { Poverty index based } \\
\text { on eight household } \\
\text { characteristics } \\
\text { Father's occupation in } \\
5 \text { categories from } \\
\text { professional to } \\
\text { unskilled } \\
\text { Parental education } \\
\text { level - age ceased } \\
\text { formal education }\end{array}$ & $\begin{array}{l}\text { Externalising } \\
\text { behaviours: associated } \\
\text { with low occupation } \\
\text { group, low parental } \\
\text { education \& poverty } \\
\text { Internalising } \\
\text { behaviours: associated } \\
\text { with low parental } \\
\text { education }\end{array}$ \\
\hline $\begin{array}{l}\text { Ingstad \& Grut, } 2007 \\
\text { Kenya }^{23}\end{array}$ & Qualitative study & $\begin{array}{l}42 \text { interviews ( } 27 \\
\text { individual; } 4 \text { group; } 11 \\
\text { secondary information) } \\
\text { including } 16 \text { children } \\
\text { in } 7 \text { strategically } \\
\text { chosen districts of } \\
\text { Kenya }\end{array}$ & $\begin{array}{l}\text { Range of disabilities } \\
\text { including physical (33), } \\
\text { intellectual/mental (9), } \\
\text { hearing (8) \& visual (5) }\end{array}$ & $\begin{array}{l}\text { Specific measures not } \\
\text { used but study does } \\
\text { examine the impact of } \\
\text { disability on the lives } \\
\text { of households with } \\
\text { disabled members } \\
\text { particularly children } \\
\text { and explores the } \\
\text { association of poverty } \\
\text { \& disability }\end{array}$ & $\begin{array}{l}\text { Complex relationship } \\
\text { between disability and } \\
\text { poverty demonstrated } \\
\text { with insights into how } \\
\text { disability and poverty } \\
\text { interact to limit life } \\
\text { chances }\end{array}$ \\
\hline $\begin{array}{l}\text { Kandamuthan, } 1997 \\
\text { India } 24\end{array}$ & $\begin{array}{l}\text { Case-control study } \\
\text { nested within Health } \\
\text { and Disability cross- } \\
\text { sectional survey of } \\
9652 \text { households in } \\
1983 \text { in an area of } \\
\text { Trivandrum, Kerala } \\
\text { State. }\end{array}$ & $\begin{array}{l}180 \text { children aged 0-14 } \\
\text { years with identified } \\
\text { disability compared } \\
\text { with } 900 \text { controls. }\end{array}$ & $\begin{array}{l}\text { Questionnaire \& clinical } \\
\text { assessment: } 8 \\
\text { outcomes studied: } \\
\text { total disabled; fits; } \\
\text { speech \& hearing } \\
\text { disability; visual } \\
\text { impairment; learning } \\
\text { disability; strange } \\
\text { behaviour; locomotor } \\
\text { disability; other }\end{array}$ & $\begin{array}{l}20 \text { SES measures used } \\
\text { but not fully stated in } \\
\text { the paper. Following } \\
\text { univariate analysis, } \\
\text { maternal education, } \\
\text { family size and } \\
\text { absence of latrine } \\
\text { retained in multi- } \\
\text { variate analysis }\end{array}$ & $\begin{array}{l}\text { Total disability } \\
\text { associated with: Low } \\
\text { maternal education - } \\
\text { adjusted OR } 2.46 \\
(1.03,5.89) ; \text { Family size } \\
>5 \text { - adjusted OR } 3.71 \\
(2.44,5.63) \text {; have latrine } \\
\text { - adjusted OR } 0.59 \\
(0.41,0.84)\end{array}$ \\
\hline $\begin{array}{l}\text { Kuklina, } 2006 \\
\text { Guatemala }\end{array}$ & $\begin{array}{l}\text { Cross-sectional study } \\
\text { nested within a birth } \\
\text { cohort including all } \\
\text { pregnancies between } \\
1996 \text { \& } 1999\end{array}$ & $\begin{array}{l}385 \text { children at } 6 \\
\text { months of age; } 342 \text { at } \\
24 \text { months of age and } \\
404 \text { at } 36 \text { months of } \\
\text { age in } 4 \text { villages in } \\
\text { Eastern Guatemala }\end{array}$ & $\begin{array}{l}\text { Neurodevelopment } \\
\text { measured using the } \\
\text { Mental Development } \\
\text { and the Psychomotor } \\
\text { Development indices } \\
\text { of the Bailey Scales of } \\
\text { Infant Development }\end{array}$ & $\begin{array}{l}\text { Maternal education } \\
\text { and household SES } \\
\text { based on household } \\
\text { characteristics and } \\
\text { possessions }\end{array}$ & $\begin{array}{l}\text { Maternal education \& } \\
\text { SES were not } \\
\text { associated with child } \\
\text { neurodevelopment }\end{array}$ \\
\hline $\begin{array}{l}\text { Loaiza \& Cappa, } \\
2005 \\
7 \text { low/middle income } \\
\text { countries }^{26}\end{array}$ & $\begin{array}{l}\text { Cross-sectional MICS2 } \\
\text { surveys in Cameroon, } \\
\text { Iraq, Jamaica, Lesotho, } \\
\text { Madagascar, Sao Tome } \\
\& \text { Principe, and } \\
\text { Suriname during the } \\
\text { period 1999-2001 }\end{array}$ & $\begin{array}{l}\text { Children 2-9 years - } \\
\text { sample sizes not stated }\end{array}$ & TQQ & $\begin{array}{l}\text { Rural v. Urban } \\
\text { Maternal education } \\
\text { Wealth quintiles }\end{array}$ & $\begin{array}{l}\text { Disability prevalence - } \\
\text { tends to be higher in } \\
\text { rural areas although } \\
\text { varies by country; } \\
\text { tends to be higher } \\
\text { among less educated } \\
\text { mothers but variable; } \\
\text { variable relationship } \\
\text { with wealth index - } \\
\text { Suriname \& } \\
\text { Madagascar show } \\
\text { social gradients in the } \\
\text { expected direction but } \\
\text { no significant } \\
\text { differences in the } \\
\text { other } 5 \text { countries }\end{array}$ \\
\hline
\end{tabular}


Table 3 Primary studies of household SEC of children with disabilities (Continued)

\begin{tabular}{|c|c|c|c|}
\hline $\begin{array}{l}\text { Meeks Gardner et al, } \\
1995 \& 1999 \\
\text { Jamaica } 27,28 \\
\text { [2 papers combined } \\
\text { as same study } \\
\text { described] }\end{array}$ & $\begin{array}{l}\text { Nested randomised } \\
\text { control trial within a } \\
\text { larger study }\end{array}$ & $\begin{array}{l}78 \text { stunted children \& } \\
26 \text { non-stunted } \\
\text { children in poor } \\
\text { neighbourhoods of } \\
\text { Kingston Jamaica - } \\
\text { data collected at } 12 \text { \& } \\
24 \text { months of age }\end{array}$ & $\begin{array}{l}\text { Mental age measured } \\
\text { using the Griffiths } \\
\text { Global Development } \\
\text { scores }\end{array}$ \\
\hline
\end{tabular}

Housing quality -
measured by quality of sanitation \& water

supply and

overcrowding

Caldwell HOME

inventory - quality of

home environment

Maternal score on

Peabody Picture

Vocabulary Test (PPVT)

of verbal reasoning

\begin{tabular}{ll}
\hline Mung'ala-Odera et al, Cross-sectional survey & 10218 out of 11416 \\
& children aged $6-9$ \\
Kenya $^{29}$ & years in the Kilifi \\
& district of Kenya (one \\
& of the poorest districts \\
& in the country - mainly \\
& subsistence farmers)
\end{tabular}

Maternal education

\section{Neurological \\ impairment measured}

in 2 phases: Phase 1 -

TQQ screen

Phase 2 - psychological

\& neurological

assessment undertaken

by trained researchers

among all screen +ve

\& similar number of

screen -ve children

Natale et al, 1992

India ${ }^{30}$
Cross-sectional survey

children aged 2-9

in households randomly sampled from 2 urban areas of Madurai, Tamil Nadu with contrasting socioeconomic characteristics

\section{TQQ screen with}

additional probe questions to ensure that only chronic conditions identified

TQQ responses grouped into 4 subscales - sensory; neuromotor; cognitive; verbal
Residence in one of 2 Overall disability: 17.4\% urban areas: one a in lowest SES area $v$

slum area with

residents of the lowest

SES (monthly family

income 10-15 US

$\$ /$ month); the other a

slightly higher SES area

(monthly family

income 32-42 US\$) $8.2 \%$ in next lowest

SES area

In logistic regression model adjusted for age, gender, birth order \& number in family OR for lowest 2.39(1.82,3.09)
Mental age at 12 \& 24 months associated maternal PPVT Not associated with housing quality or HOME score

Moderate/severe

in impairment NOT associated with any of the SES measures on univariate analysis

All subtypes except verbal significantly higher in lowest SES area

\begin{tabular}{|c|c|c|c|c|c|}
\hline $\begin{array}{l}\text { Rischewski, } 2008 \\
\text { Rwanda }^{31}\end{array}$ & $\begin{array}{l}\text { Nationwide matched } \\
\text { case-control study } \\
\text { (adults \& children) } \\
\text { nested within a } \\
\text { national cross-sectional } \\
\text { survey }\end{array}$ & $\begin{array}{l}93 \text { cases aged }<15 \text { yrs } \\
\text { identified in the } \\
\text { national survey } \\
\text { matched for age \& } \\
\text { gender with } 146 \\
\text { controls }\end{array}$ & $\begin{array}{l}\text { Musculoskeletal } \\
\text { impairment (MSI) } \\
\text { ranging from knock } \\
\text { knees to quadriplegia }\end{array}$ & $\begin{array}{l}\text { Per capita household } \\
\text { expenditure; } x 2 \\
\text { household asset } \\
\text { possession measures }\end{array}$ & $\begin{array}{l}\text { MSI in children }<15 \\
\text { NOT associated any of } \\
\text { the household poverty } \\
\text { measures }\end{array}$ \\
\hline $\begin{array}{l}\text { Shawky, } 2002 \\
\text { Saudi Arabia } 32\end{array}$ & $\begin{array}{l}\text { Case-control study } \\
\text { based on four cross- } \\
\text { sectional cohorts }\end{array}$ & $\begin{array}{l}1225 \text { children aged 6- } \\
20 \text { years with } \\
\text { disabilities identified } \\
\text { from specialist centres } \\
\text { in Jeddah and } 3405 \\
\text { non-disabled school } \\
\text { children sampled from } \\
42 \text { boys' and } 42 \text { girls' } \\
\text { schools }\end{array}$ & $\begin{array}{l}\text { Auditory, visual \& } \\
\text { mental impairment }\end{array}$ & $\begin{array}{l}\text { Maternal education; } \\
\text { maternal working } \\
\text { status }\end{array}$ & $\begin{array}{l}\text { No maternal education } \\
\text { associated with: } \\
\text { auditory impairment } \\
\text { OR } 13.3 \text { (7.2,27.8); } \\
\text { visual impairment OR } \\
3.7(2.1,6.6) ; \text { mental } \\
\text { impairment OR } 5.5 \\
\text { (3.8,8.1) - all adjusted } \\
\text { for maternal age at } \\
\text { birth, parity, working } \\
\text { status, consanguinity \& } \\
\text { multiparity }\end{array}$ \\
\hline $\begin{array}{l}\text { Suris \& Blum, } 1993 \\
>20 \text { countries - high } \\
\text { \& low income }\end{array}$ & $\begin{array}{l}\text { Secondary data } \\
\text { analysis of cross- } \\
\text { sectional surveys }\end{array}$ & $\begin{array}{l}\text { 10-14 yr olds in } 19 \\
\text { countries and } 15-19 \text { yr } \\
\text { olds in } 23 \text { countries - } \\
\text { only rates calculated - } \\
\text { no numbers given. } \\
\text { Data derived from UN } \\
\text { International Disability } \\
\text { Statistics Database } \\
\text { (DISTAT) }\end{array}$ & $\begin{array}{l}\text { No specific definition } \\
\text { of disability stated - } \\
\text { total disability rates as } \\
\text { reported to UN }\end{array}$ & $\%$ female illiteracy & $\begin{array}{l}\text { \% country level female } \\
\text { illiteracy not correlated } \\
\text { with disability rates for } \\
\text { adolescents in either } \\
\text { age group }\end{array}$ \\
\hline
\end{tabular}


Table 3 Primary studies of household SEC of children with disabilities (Continued)

\begin{tabular}{|c|c|c|}
\hline $\begin{array}{l}\text { Izutsu et al, } 2006 \\
\text { Bangladesh }^{34}\end{array}$ & Cross-sectional survey & $\begin{array}{l}\text { Random samples of } \\
187 \text { boys \& } 137 \text { girls } \\
\text { from non-slum areas } \\
\text { and } 157 \text { boys and } 121 \\
\text { girls from slum areas } \\
\text { of Dhaka aged } 11-18 \\
\text { years }\end{array}$ \\
\hline
\end{tabular}

$\begin{array}{ll}\text { the Youth Self Report } & \\ & \text { questionnaire } \\ \text { administered in their } & \text { homes by trained } \\ \text { interviewers }\end{array}$

\begin{tabular}{|c|c|c|c|c|c|}
\hline $\begin{array}{l}\text { Thomas, } 2005 \\
\text { Cambodia }^{35}\end{array}$ & Qualitative study & $\begin{array}{l}\text { Key informant } \\
\text { interviews at } 3 \text { centres } \\
\text { for disabled persons in } \\
\text { Cambodia - interviews } \\
\text { with staff and } \\
\text { administrators; focus } \\
\text { group interviews - one } \\
\text { with } 13 \text { disabled adults } \\
\text { \& one with } 4 \text { disabled } \\
\text { children; home visits } \\
\text { and interviews with } \\
\text { four disabled adults } \\
\text { and four disabled } \\
\text { children \& their } \\
\text { parents }\end{array}$ & $\begin{array}{l}\text { Range of different } \\
\text { disabilities }\end{array}$ & Poverty & $\begin{array}{l}\text { Poverty identified as } \\
\text { both cause and } \\
\text { consequence of } \\
\text { disability - discussion } \\
\text { of mechanisms by } \\
\text { which poverty impacts } \\
\text { on disability and vice } \\
\text { versa based on } \\
\text { qualitative data }\end{array}$ \\
\hline $\begin{array}{l}\text { Thomas, } 2005 \\
\text { India }^{36}\end{array}$ & Qualitative study & $\begin{array}{l}\text { Field visits to } 7 \text { centres } \\
\text { for disabled persons. } \\
\text { Focus group interviews } \\
\text { with } 27 \text { at one centre } \\
\text { \& } 12 \text { at another centre. } \\
\text { Small number of } \\
\text { individual interviews } \\
\text { with disabled persons } \\
\text { or parents of disabled } \\
\text { children }\end{array}$ & $\begin{array}{l}\text { Range of different } \\
\text { disabilities }\end{array}$ & Poverty & $\begin{array}{l}\text { Poverty identified as } \\
\text { both cause and } \\
\text { consequence of } \\
\text { disability - discussion } \\
\text { of mechanisms by } \\
\text { which poverty impacts } \\
\text { on disability and vice } \\
\text { versa based on } \\
\text { qualitative data }\end{array}$ \\
\hline $\begin{array}{l}\text { Thomas, } 2005 \\
\text { Rwanda }^{37}\end{array}$ & Qualitative study & $\begin{array}{l}\text { Key informant } \\
\text { interviews at } 3 \text { centres } \\
\text { for disabled persons in } \\
\text { Rwanda - } 2 \text { focus } \\
\text { group interviews ( } 27 \\
\text { and } 20 \text { disabled } \\
\text { persons) and } 4 \\
\text { individual interviews. } \\
\text { No specific reference } \\
\text { to interviews with } \\
\text { children or parents of } \\
\text { children. }\end{array}$ & $\begin{array}{l}\text { Range of different } \\
\text { disabilities }\end{array}$ & Poverty & $\begin{array}{l}\text { Poverty identified as } \\
\text { both cause and } \\
\text { consequence of } \\
\text { disability - not specific } \\
\text { to children }\end{array}$ \\
\hline $\begin{array}{l}\text { UNICEF, } 2008 \\
18 \text { low/middle } \\
\text { income countries }^{2}\end{array}$ & $\begin{array}{l}\text { MICS3 cross-sectional } \\
\text { surveys in Albania, } \\
\text { Bangladesh, Belize, } \\
\text { Bosnia and } \\
\text { Herzegovina, } \\
\text { Cameroon, Central } \\
\text { African Republic, } \\
\text { Georgia, Ghana, } \\
\text { Mauritania, Mongolia, } \\
\text { Montenegro, Sao } \\
\text { Tome \& Principe, } \\
\text { Serbia, Sierra Leone, } \\
\text { Suriname, Thailand, } \\
\text { TYFR Macedonia and } \\
\text { Uzbekistan during the } \\
\text { period 2005-2008 }\end{array}$ & $\begin{array}{l}\text { Children aged } 2-9 \\
\text { years ranging from } \\
1,537 \text { children in Belize } \\
\text { to } 58,441 \text { in } \\
\text { Bangladesh }\end{array}$ & TQQ screening & $\begin{array}{l}\text { Household wealth } \\
\text { index (60\% poorest v. } \\
40 \% \text { richest) } \\
\text { Maternal education } \\
\text { Rural/urban }\end{array}$ & $\begin{array}{l}\text { Wealth index: only in } 6 \\
\text { countries (Bangladesh, } \\
\text { Georgia, Mongolia, } \\
\text { Serbia, Sierra Leone \& } \\
\text { Thailand were disabled } \\
\text { children at greater risk } \\
\text { of living in the poorest } \\
60 \% \text { of households } \\
\text { Low maternal } \\
\text { education: only } 6 \\
\text { countries show a } \\
\text { greater risk for } \\
\text { disabled children of } \\
\text { living in households } \\
\text { with mothers with low } \\
\text { education } \\
\text { Rural v. Urban: very } \\
\text { little association of } \\
\text { disability with rural } \\
\text { living }\end{array}$ \\
\hline
\end{tabular}


Table 3 Primary studies of household SEC of children with disabilities (Continued)

\begin{tabular}{|c|c|c|c|c|c|}
\hline $\begin{array}{l}\text { VanLeit et al, } 2007 \\
\text { Cambodia }^{38}\end{array}$ & Cross-sectional survey & $\begin{array}{l}500 \text { children } 0-18 \text { years } \\
\text { with disabilities - no } \\
\text { controls included in } \\
\text { the study }\end{array}$ & $\begin{array}{l}\text { Full range of } \\
\text { disabilities - objective } \\
\text { was to identify the } \\
\text { functional status of } \\
\text { disabled children in } \\
\text { Cambodia }\end{array}$ & Poverty (< $1 \$ /$ day) & $\begin{array}{l}49 \% \text { of households } \\
\text { identified in the survey } \\
\text { were living in poverty }\end{array}$ \\
\hline
\end{tabular}

with disability in LAMI countries. As shown in Table 3 the qualitative data describe a close association of disability (both adult and child) with poverty; however, the association is not reported consistently in the quantitative studies. Potential mechanisms by which poverty and low household SEC may be both a cause and consequence of disability are outlined in the reviews and the qualitative studies. The reviews identified the living conditions of poor people in LAMI countries as a primary causal mechanism. Preventable impairments associated with communicable, maternal and perinatal disease and injuries [40], and malnutrition in childhood [39] were identified as key mechanisms by which poverty caused disability. The reviews (see particularly Mitra [41]) presented socially plausible mechanisms by which disability itself both in adults and children causes poverty and exacerbates existing poverty. Baylies [43] and Dudzik et al [44] identified social structures, war and social unrest which have the greatest impact on the poor as mechanisms linking poverty and disability. Smith [46] reviewing hearing impairment in developing countries, identified poor preventive health services as a factor in high prevalence of deafness and hearing impairment among children due to perinatal factors, chronic otitis media and ototoxic drugs.

The qualitative data provided further support for the role of poverty as both cause and consequence of disability. Ingstad and Grut [23], based on case studies derived from their fieldwork in Kenya, identified congenital conditions, conditions occurring in pregnancy and childbirth, malaria and epilepsy as mechanisms through which poverty leads to disability. All five reports contained rich data from their case studies illustrating how disability exacerbates and precipitates poverty.

Of the 8 studies reporting total childhood disability rates by household SEC, three were single country studies [24,30,38]. In Kandamuthan's case-control study [24] disabled children aged 0-14 years were more than twice as likely to have a mother with low education as their non-disabled peers. Natale et al's study [30] reported a more than twofold increase in prevalence of disability (17.4\%) among children aged 2-9 years among those living in the lowest socioeconomic area compared with children living in a slightly more advantaged but still poor area (8.2\%). Although VanLeit et al [38] report $49 \%$ of the children aged $0-18$ years in their study living in poverty, their study has no control group so the significance of this finding is difficult to interpret. By contrast, there is no consistent association of total childhood disability with household SEC reported by the five multi-country studies $[2,4,30,26,33]$. Six out of the nine countries included in Filmer's study [20] show significant difference in prevalence of childhood disability by household SEC although only one, Indonesia, shows a clear social gradient. However, in the same author's 2008 study [4], in only two out of 13 countries, Indonesia and India, were significant differences between rich and poor households found. The studies by Loaiza \& Cappa [26] and UNICEF [2], based on the Multi-Indicator Cluster Surveys 2 (MICS2) and 3 (MICS3) respectively, report variable findings with only a few countries showing significant relationships of total disability among 2-9 year old children with poorer household SEC. In their study, Suris and Blum [33] use United Nations disability data from both high and low/middle income countries to examine the association of countrylevel prevalence of disability among adolescents aged 15-19 years. They found no correlation of country level female illiteracy with prevalence of disability in this age group.

The six studies that examined the association of child mental illness, behavioural problems and mental retardation with household SEC [16,17,19,22,32,34] all reported significant relationships with poor household SEC. Bashir et al [17], Durkin et al [19] and Shawky [32] reported a strong association of mild mental retardation with household SEC. Durkin et al [19] reported a strong association of severe mental retardation (IQ < 50) with no maternal education and rural living. Anselmi et al [16], in a prospective cohort study, found a significant association of externalising and internalising behaviours, and attention problems at age 12 years with low family income at 4 years of age. Hackett et al [22] reported similar findings in a cross-sectional survey. Izutsu et al [34] found a significant association of residence in a slum area with conduct problems but not with other behavioural difficulties.

None of the studies reporting on the association of neurological, neurodevelopmental and musculoskeletal problems with household SEC [25,27-29,31] found any significant association. Shawky [32] in a case-control study, found a very high odds ratio (13.3(95\% CI 
7.2,27.8)) of children and young people aged 6-20 years with hearing loss having a mother with no education. Bastos et al [18] reported a two-fold increase in prevalence of hearing loss within the speech frequency range in urban areas (37\%) compared with rural areas (18\%) of northern Tanzania. The only study reporting on visual impairment [32] found a significant association with no maternal education.

The quality of included studies was variable. Table 4 summarises the quality assessment of the quantitative studies using the criteria listed in Table 2. Seventeen of the 19 quantitative studies had a medium or low risk of bias. The main sources of potential bias were single cross-sectional survey design and high or unreported non-response rates. Two studies had high risk of bias: Suris and Blum [33] as the study was based on national disability rates with no information on definitions, no information of how data were collected and only national level female illiteracy rates as a measure of socioeconomic status; VanLeit et al [38] as the study included only adolescents with disabilities with no comparison or control group.

The methodologies used in the country-based reports to collect qualitative data, while enabling rich data to be collected, had weaknesses related to inadequately described sampling methods and limited explanation of how themes and case studies were identified, selected and analysed. The samples included both adults and children limiting the validity of the findings for studying childhood disability.

\section{Discussion}

To our knowledge, this is the first review specifically reporting on the available literature on the association of childhood disability with home socio-economic circumstances in LAMI countries. We identified primary quantitative and qualitative studies and reviews that specifically addressed the household SEC of disabled children in LAMI countries. As this is a narrative review, we have not carried out meta-analysis of results of empirical quantitative studies.

\section{Main findings}

This review has shown that evidence from available literature on the association of childhood disability and household SEC in LAMI countries is inconsistent and inconclusive. This is likely to be due to differences in: measures of household SEC used in the studies; definitions of disability; outcomes studied; study design and methods. Some measures of household SEC, for example, residence in urban versus rural areas and female illiteracy rates are likely to be less precise than those based on individual household SEC measures. Studies using more than one measure of household SEC are more likely to identify associations with childhood disability than those using single measures as different measures may capture different pathways and mechanisms associated with the outcomes. For example, mild learning disability (termed mental retardation in the included studies) is likely to be associated with household education level while hearing impairment is likely to be associated with poor perinatal care and lack of preventive health care secondary to family poverty.

The definition of disability has developed in recent decades and the International Classification of Functioning, Disability and Health promotes a bio-psycho-social model that incorporates components of the medical and social models [10]. Disability arises out of the interaction between health conditions with contextual factors including the environment (SEC is part of this) and personal factors. With the United Nations Convention on the Rights of Persons with Disability, disability is becoming a rights based issue and its definition is changing [11]. However the literature described in this paper defines disability in terms of impairments.

The association of poor household SEC with learning disability and behaviour problems was the most consistent finding of the review. All six studies reported a positive association of poor household SEC with these outcomes and all these studies had a low or medium risk of bias. By contrast, studies with neurodevelopmental and neuromuscular problems as the outcome reported no association with poor household SEC. These studies also carried a low or medium risk of bias. One possible explanation for this differential association is that children with physical or neurological problems die prematurely and so are not available for counting whereas children with learning disability or behavioural problems survive; another explanation is that the neurodevelopmental and neuromuscular conditions may be more likely to have a genetic origin not associated with SEC.

Only two studies reported on hearing loss as the outcome of interest. They reported strikingly different associations with SEC. These divergent findings may be partly explained by the different measures of household SEC used.

The studies in which overall disability was the outcome of interest also reported divergent findings. The multi-country studies, based on secondary analysis of the UNICEF Multiple Indicator Cluster Surveys (MICS), Demographic and Health Surveys and other survey datasets, reported a positive association of childhood disability rates with poor household SEC in a few countries but no association in the majority of countries studied. Children with disability were identified using the TTQ in several of these studies. This instrument was designed with two stages; the questionnaire and then a clinical 
Table 4 Quality assessment of quantitative studies

\begin{tabular}{|c|c|c|c|c|c|c|c|c|c|}
\hline Study & Design & Purpose & $\begin{array}{l}\text { Data } \\
\text { collection } \\
\text { methods }\end{array}$ & $\begin{array}{l}\text { Sampling \& } \\
\text { sample size }\end{array}$ & Definitions & $\begin{array}{l}\text { Response } \\
\text { rate }\end{array}$ & $\begin{array}{l}\text { Measures of home } \\
\text { circumstance \&/or } \\
\text { SES }\end{array}$ & $\begin{array}{l}\text { Child } \\
\text { data }\end{array}$ & $\begin{array}{l}\text { Overall } \\
\text { risk of } \\
\text { bias }\end{array}$ \\
\hline $\begin{array}{l}\text { Anselmi et al, } \\
2008^{16} \\
\text { Brazil }\end{array}$ & Optimum & Adequate & Optimum & Optimum & Optimum & Optimum & Adequate & Optimum & Low \\
\hline $\begin{array}{l}\text { Bashir et al, } \\
2002 \\
\text { Pakistan }^{17}\end{array}$ & Optimum & Optimum & Optimum & Optimum & Optimum & Adequate & Adequate & Optimum & Low \\
\hline $\begin{array}{l}\text { Bastos et al, } \\
1995 \\
\text { Tanzania }^{18}\end{array}$ & Adequate & Optimum & Optimum & Optimum & Optimum & Optimum & $\begin{array}{l}\text { Least valuable (urban } \\
\text { v. Rural) }\end{array}$ & Optimum & Medium \\
\hline $\begin{array}{l}\text { Durkin et al, } \\
1998 \\
\text { Pakistan }\end{array}$ & Adequate & Optimum & Optimum & Optimum & Optimum & Optimum & Adequate & Optimum & Low \\
\hline $\begin{array}{l}\text { Filmer } 2005 \\
9 \text { low \& middle } \\
\text { income } \\
\text { countries }^{20}\end{array}$ & Adequate & Adequate & Optimum & Optimum & Optimum & $\begin{array}{l}\text { Least } \\
\text { valuable }\end{array}$ & Adequate & Optimum & Medium \\
\hline $\begin{array}{l}\text { Filmer } 2008 \\
13 \text { low \& middle } \\
\text { income } \\
\text { countries }^{4} \\
\text { [NB: some } \\
\text { overlap with }^{\text {Filmer 2005] }} \\
\end{array}$ & Adequate & Adequate & Optimum & Optimum & Optimum & $\begin{array}{l}\text { Least } \\
\text { valuable }\end{array}$ & Adequate & Optimum & Medium \\
\hline $\begin{array}{l}\text { Hackett et al, } \\
1999 \\
\text { India }^{22}\end{array}$ & Adequate & Optimum & Optimum & Optimum & Optimum & Adequate & Optimum & Optimum & Low \\
\hline $\begin{array}{l}\text { Kandamuthan, } \\
1997 \\
\text { India }^{24}\end{array}$ & Adequate & Optimum & Optimum & Optimum & Optimum & Adequate & Optimum & Optimum & Low \\
\hline $\begin{array}{l}\text { Kuklina, } 2006 \\
\text { Guatemala }^{25}\end{array}$ & Adequate & Optimum & Optimum & Adequate & Optimum & Adequate & Optimum & Optimum & Medium \\
\hline $\begin{array}{l}\text { Loaiza \& Cappa, } \\
2005 \\
7 \text { low/middle } \\
\text { income } \\
\text { countries }^{26}\end{array}$ & Adequate & Adequate & Optimum & Optimum & Optimum & $\begin{array}{l}\text { Least } \\
\text { valuable }\end{array}$ & Optimum & Optimum & Medium \\
\hline $\begin{array}{l}\text { Meeks Gardner } \\
\text { et al, } 1995 \& \\
1999 \\
\text { Jamaica } \\
\text { [2 papers } 28 \\
\text { combined as } \\
\text { same study } \\
\text { described] }\end{array}$ & Adequate & Adequate & Optimum & Adequate & Optimum & Optimum & Adequate & Optimum & Medium \\
\hline $\begin{array}{l}\text { Mung'ala-Odera } \\
\text { et al, } 2006 \\
\text { Kenya }^{29} \\
\end{array}$ & Adequate & Optimum & Optimum & Optimum & Optimum & Adequate & Adequate & Optimum & Medium \\
\hline $\begin{array}{l}\text { Natale et al, } \\
1992 \\
\text { India }^{30}\end{array}$ & Adequate & Optimum & Optimum & Adequate & Optimum & Optimum & Least valuable & Optimum & Medium \\
\hline $\begin{array}{l}\text { Rischewski, } 2008 \\
\text { Rwanda }^{31}\end{array}$ & Adequate & Optimum & Optimum & Adequate & Adequate & Optimum & Adequate & Optimum & Low \\
\hline $\begin{array}{l}\text { Shawky, } 2002 \\
\text { Saudi Arabia } \\
\text { 32 }\end{array}$ & Adequate & Optimum & Optimum & Optimum & Optimum & Optimum & Adequate & Optimum & Low \\
\hline $\begin{array}{l}\text { Suris \& Blum, } \\
1993 \\
>20 \text { countries - } \\
\text { high \& low } \\
\text { income }\end{array}$ & $\begin{array}{l}\text { Least } \\
\text { valuable }\end{array}$ & Optimum & Adequate & $\begin{array}{l}\text { Least valuable } \\
\text { - only national } \\
\text { rates given }\end{array}$ & $\begin{array}{l}\text { Least valuable - } \\
\text { no information on } \\
\text { definitions used }\end{array}$ & $\begin{array}{l}\text { Least } \\
\text { valuable }\end{array}$ & $\begin{array}{l}\text { Least valuable - only } \\
\text { national level of } \\
\text { female illiteracy given }\end{array}$ & $\begin{array}{l}\text { Least } \\
\text { valuable }\end{array}$ & High \\
\hline
\end{tabular}


Table 4 Quality assessment of quantitative studies (Continued)

\begin{tabular}{|c|c|c|c|c|c|c|c|c|c|}
\hline $\begin{array}{l}\text { Izutsu al, } 2006 \\
\text { Bangladesh }^{34}\end{array}$ & Adequate & Optimum & Optimum & Adequate & Optimum & Optimum & Least valuable & Optimum & Medium \\
\hline $\begin{array}{l}\text { UNICEF, } 2008 \\
18 \text { low/middle } \\
\text { income } \\
\text { countries }^{2}\end{array}$ & Adequate & Adequate & Optimum & Optimum & Optimum & $\begin{array}{l}\text { Least } \\
\text { valuable }\end{array}$ & Adequate & Optimum & Medium \\
\hline $\begin{array}{l}\text { VanLeit et al, } \\
2007 \\
\text { Cambodia }^{38}\end{array}$ & $\begin{array}{l}\text { Least } \\
\text { valuable }\end{array}$ & Optimum & Adequate & Adequate & Optimum & Optimum & Adequate & Optimum & High \\
\hline
\end{tabular}

assessment of children who scored positively on the questionnaire. Unfortunately the clinical assessment is often not performed, making the TQQ invalid. One of these studies [33] had a high risk of bias; the other four studies had a medium risk of bias. A positive association of childhood disability rates with poor household SEC was reported by the three studies that collected and analysed primary data; however, one of these studies [38] had a high risk of bias as it was based on a case series with no control or comparison group.

The reviews identified by the search strategy reported socially and biologically plausible mechanisms by which poverty might be both a cause and consequence of disability. They identified the living conditions of poor people in LAMI countries as a primary causal mechanism; as Elwan [40] states "disability in developing countries stems largely from preventable impairments associated with communicable, maternal and perinatal disease and injuries". Malnutrition was identified as a specific cause of disability in childhood [39]. The reviews (see particularly Mitra [41]) presented socially plausible mechanisms by which disability causes poverty and exacerbates existing poverty.

The qualitative data from country-based reports had methodological weaknesses that limited the validity of their findings in relation to the association of poor household SEC with childhood disability. However, the studies provided further support for the role of poverty as both cause and consequence of disability. Ingstad and Grut [23], based on case studies derived from their fieldwork in Kenya, identified congenital conditions, conditions occurring in pregnancy and childbirth, malaria and epilepsy as mechanisms through which poverty leads to disability. All five studies reported rich data from their case studies illustrating how disability exacerbates and precipitates poverty.

\section{Comparison with literature from high income countries and other literature from LAMI countries}

To our knowledge, no equivalent review of the association of childhood disability with poor home circumstances/low SES in high income countries has been published. There is a substantial literature from the UK (for example, Gordon et al [47]; Blackburn et al 2010 [48], Emerson et al [49]), the USA (for example, Newacheck [50]; Newacheck and Halfon [51]) Australia (for example, Bor et al [52]; Leonard et al [53]) and Scandinavia (for example, Hjern et al [54]; Berntsson and Kohler [55]) showing that childhood disability is associated with higher risk of living in a poor/low income household. Gordon et al [47] assert that poverty in high income countries is both cause and consequence of childhood disability but we are not aware of specific studies confirming that poverty is causally related to disability in childhood.

The findings of this review are consistent with the literature in high income countries in that poverty is viewed as both cause and consequence of childhood disability; however, although there is strong evidence in both high income and LAMI countries for poverty as a consequence of disability, there are no empirical studies demonstrating the causal relationship of poverty to disability. Whereas studies from high income countries show a consistent association of childhood disability with poor home circumstances/low SES, the quantitative studies reviewed here show a much less consistent association. This is likely to result from differences in definition of disability, in data quality and sampling methods.

Three quantitative studies [56-58] not included in this review as they did not analyse child and adult disability separately, reported lower incomes and fewer assets among households with disabled members (adults and/ or children) and those with no disabled members in three sub-Saharan African countries (Namibia, Malawi and Zimbabwe). Their findings indicate that the use of rigorous research methodology with attention to sampling, disability definition, and detailed measurement of household SEC can result in very similar findings in different countries.

\section{Limitations}

Publication bias is a possible threat to the conclusions of this review. Although we aimed to identify a broad range of journal papers and reports on childhood disability, we did not include a search of grey literature, hand-searching of key journals or advice from key 
informants. As a consequence, the review is unlikely to include all available studies of the links between household SEC and childhood disability in LAMI countries. We used general search terms for disability, did not include specific diagnoses and chose to limit the search to publications from 1990 onwards in order to exclude studies with outdated information and to limit the volume of literature identified. This may have led to significant omissions from the review.

\section{Implications for further study}

The reviews and qualitative studies included in this review suggest that poverty is both a cause and consequence of disability in childhood. They outline biologically and socially plausible mechanisms by which poverty may exert its influence. By contrast, the empirical studies reviewed here report an inconsistent association of household SEC with childhood disability. Further research in this area is essential to clarify the relationship. The findings of this review, and those of Maulik and Darmstadt [3] indicate the need to address the following issues in future research into the association of childhood disability with household SEC:

Definitions of disability - although many of the studies reviewed here clearly stated the definitions used, there is a need, as Maulik and Darmstadt [3] point out, for consistency and standardisation of the definitions used and awareness of the problems of definition that impose limitations on conclusions that can be drawn

Types of disability - this review is consistent with Maulik and Darmstadt's [3] observation that the majority of studies focus on intellectual impairment and overall disability rates. Future studies could focus on other relatively common disabling conditions such as cerebral palsy. We found no studies using functional definitions of disability, such as defined in the ICF-CY - these would provide further valuable insights into the association with household SEC

Measures of household SEC - the review demonstrates that more than one measure of household SEC is necessary to study comprehensively the association with childhood disability as different measures such as maternal education and household wealth may have different associations with disability.

Study design - cross-sectional surveys, the most common design among the quantitative studies reviewed here, are relatively cheap to organise and are valuable for descriptive epidemiology. Further study to describe the relationship of childhood disability and household SEC will be valuable and cross-sectional surveys will continue to have a role. However, such surveys are only able to show associations; longitudinal prospective studies are necessary to comment on causal mechanisms particularly as relates to poverty as a cause rather than consequence of disability.

Mixed methods studies - the review shows that the qualitative studies provide valuable insights into possible social and biological mechanisms by which poverty might impact on disability. Future research would be strengthened by a mixed methods design that combined rigorous qualitative research preferably nested within a well-designed prospective quantitative study.

\section{Conclusion}

This review indicates that, despite socially and biologically plausible mechanisms underlying the association of low household SEC with childhood disability in LAMI countries, the empirical evidence from quantitative studies is inconsistent and contradictory. There is an urgent need for a more robust evidence base to inform the development of effective health and social policies aimed at reducing the burden of childhood disability in LAMI countries.

\section{List of abbreviations}

ICF-CY: International Classification of Functioning, Disability and Health Children and Young People; IMF: International Monetary Fund; MICS:

Multiple Indicator Cluster Survey; LAMI: Low and middle income; Paris21: Partnerships in Statistics for Development in the $21^{\text {st }}$ century; POPLINE:

Population information online; SEC: socio-economic circumstances; SIMPOC: Statistical Information and Monitoring Programme on Child Labour; SINTEF: The Foundation for Scientific and Industrial Research; TQQ: Ten Questions Questionnaire; UNCRPD: United Nations Convention on the Rights of Persons with Disabilities; UNICEF: United Nations Children's Fund; WHO: World Health Organization.

\section{Acknowledgements and funding}

We thank the Institute of Advanced Studies (IAS) at the University of Warwick for funding that facilitated this research project. The IAS had no role in the study design; analysis and interpretation of data; in the writing of the manuscript; or in the decision to submit this manuscript for publication.

\section{Author details}

${ }^{1}$ Health Sciences Research Institute, Warwick Medical School, University of Warwick, (Gibbet Hill Road), Coventry, (CV4 7AL), UK. ${ }^{2}$ School of Health and Social Studies, University of Warwick, (Gibbet Hill Road), Coventry, (CV47 $1 \mathrm{GN})$, UK. ${ }^{3} \mathrm{NHS}$ Kidney Care, New Croft House, (Market Street East), Newcastle upon Tyne, (NE1 6ND), UK. ${ }^{4}$ School of Health and Social Studies, University of Warwick, (Gibbet Hill Road), Coventry, (CV47 1GN), UK. ${ }^{5}$ School of Health and Social Studies, University of Warwick, (Gibbet Hill Road), Coventry, (CV47 1GN), UK.

\section{Authors' contributions}

DS critically appraised the papers and helped to draft the manuscript. CB conceived of the study and participated in its design and coordination. FM conducted the literature search. JR conceived of the study and participated in its design and coordination NS critically appraised the papers and drafted the manuscript. All authors read and approved the final manuscript.

\section{Competing interests}

The authors declare that they have no competing interests.

Received: 4 August 2011 Accepted: 21 December 2011 Published: 21 December 2011

\section{References}

1. World Health Organization: World Report on Disability Geneva: WHO; 2011. 
2. Univeristy of Wisconsin and UNICEF: Monitoring Child Disability in Developing Countries: Results from the Multiple Indicator Cluster Surveys New York: UNICEF; 2008

3. Maulik PK, Darmstadt GL: Childhood disability in low-and middle-income countries: Overview of screening, prevention, services, legislation and epidemiology. Pediatrics 2007, 120(suppl):1-55.

4. Filmer D: Disability, poverty, and schooling in developing countries: Results from 14 household surveys. The World Bank Economic Review 2008, 141-163.

5. Mont D: Measuring disability prevalence. Social protection discussion paper No 0706 Washington: The World Bank; 2007.

6. Bickenbach J, Chatterji S, Badley E, Ustin T: Models of disablement, universalisim and the international classification of impairments, disabilities and handicaps. Social Science and Medicine 1999, 48(9):1173-1187

7. Wasserman D: Philosophical issues in the definition and social response to disability. In Handbook of Disability Studies. Edited by: Seelman GK, Bury M. Thousand Oaks, California: Sage Publications; 2001:.

8. Colver A, Dickinson H, SPARCLE group: Study Protocol: Determinants of participation and quality of life of adolescents with cerebral palsy: a longitudinal study (SPARCLE 2). BMC Public Health 2010, 10:208.

9. MCDougall J, Wright $V$, Rosenbaum R: The ICF model of functioning and disability: incorporating quality of life and human development. Developmental Neurorehabilitiation 2010, 13(3):204-211.

10. World health Organization: International Classification of Functioning Disability and Health - Children and Youth Version Geneva: WHO; 2007.

11. United Nations Conventions on the Rights of Persons with Disabilities. [http://www.un.org/disabilities].

12. Gottlieb C, Maenner M, Cappa C, Durkin M: Child disability screening, nutrition, and early learning in 18 countries with low and middle incomes: data from the third round of UNICEF's Multiple Indicator Cluster Survey (2005-06). Lancet 2009, 374(9704):1831-1839.

13. Davidson L, Durkin MS, Khan NZ: Studies of children in developing countries. How soon can we prevent neurodisability in childhood? Developmental Medicine and Child Neurology 2003, 45(Suppl 96):18-24

14. Msall ME, Hogan DP: Counting Children With Disability in Low-Income Countries: Enhancing Prevention, Promoting Child Development, and Investing in Economic Wellbeing. Pediatrics 2007, 120:182-185.

15. Muñoz B, West S: Blindness and visual impairment in the Americas and the Caribbean. British Journal of Ophthalmology 2002, 86:498-504.

16. Anselmi $L$, Barros FC, Teodoro ML, Piccinini CA, Menezes AMB, Araujo CL, Rohde LA: Continuity of behavioral and emotional problems from preschool years to pre-adolescence in a developing country. Child Psychology and Psychiatry 2008, 49(5):499-507.

17. Bashir A, Yagoob M, Ferngren $H$, Gustavson $K$, Rydelius $P$, Ansari $T$, Zaman S: Prevalence and associated impairments of mild mental retardation in six- to ten-year old children in Pakistan: A prospective study. Acta Paediatrica 2002, 91(7):833-837.

18. Bastos I, Mallyab J, Ingvarssona L, Reimerc $\AA$, Andréasson L: Middle ear disease and hearing impairment in northern Tanzania: A prevalence study of school children in the Moshi and Monduli districts. International Journal of Pediatric Otorhinolaryngology 1995, 32:1-12.

19. Durkin M, Hasan Z, Hasan K: Prevalence and correlates of mental retardation among children in Karachi, Pakistan. American Journal of Epidemiology 1998, 147(3):281-288.

20. Filmer D: Disability, poverty and schooling in developing countries: Results from 11 household surveys. Social Protection Washington DC: The World Bank; 2005

21. Grut $\mathrm{L}$, Ingstad $\mathrm{B}$ : This is my life - Living with a disability in Yemen: $A$ qualitative study Oslo: SINTEF Health Research; 2006.

22. Hackett $R$, Hackett $L$, Bhakta $P$, Gowers S: The prevalence and associations of psychiatric disorder in children in Kerala, South India. Journal of Child Psychology and Psychiatry 1999, 40(5):801-807.

23. Ingstad B, Grut L: See me, and do not forget me: People with disabilities in Kenya Oslo: SINTEF Health Research; 2007.

24. Kandamuthan M: Socio-economic factors and childhood disability in Trivandrum of South India. International Journal of Rehabilitation Research 1997, 20:335-339.

25. Kuklina EV, Ramakrishnan U, Stein AD, Barnhart HH, Martorell R: Early childhood growth and development in rural Guatemala. Early Human Development 2006, 82:425-433.
26. Loaiza E, Cappa C: Measuring children's disability via household surveys: The MICS experience. Population Association of America Philadelphia, PA; 2005, 27.

27. Meeks Gardner JM, Grantham-McGregor SM, Himes J, Chang S: Activity and behavioral development in stunted and nonstunted children and response to nutritional supplementation. Child Development 1995, 66:1785-1797.

28. Meeks Gardner JM, Grantham-McGregor SM, Himes J, Chang S: Behaviour and development of stunted and nonstunted Jamaican children. Journal of Child Psychology and Psychiatry 1999, 40(5):819-827.

29. Mung'ala-Odera V, Meehan R, Njuguna P, Mturi N, Alcock K, Newton C: Prevalence and risk factors of neurological disability and impairment in children living in rural Kenya. International Journal of Epidemiology 2006, 35:683-688.

30. Natale JE, Joseph JG, Bergen R, Thulasiraj R, Rahmathulla L: Prevalence of childhood disability in a Southern Indian city: Independent effect of small differences in social status. International Journey of Epidemiology 1992, 21(2):367.

31. Rischewski D, Kuper $H$, Atijosan O, Simms V, Jofret-Bonet M, Foster A, Lavy C: Poverty and musculoskeletal impairment in Rwanda. Transactions of the Royal Society of Tropical Medicine and Hygiene 2008, 102:608-617.

32. Shawky S, Abalkhail B, Soliman N: An epidemiological study of childhood disability in Jeddah, Saudi Arabia. Paediatric and Perinatal Epidemiology 2002, 16:61-66.

33. Suris J-C, Blum RW: Disability rates among adolescents: An international comparison. Journal of Adolescent Health 1993, 14:548-552.

34. Izutsu T, Tsutsumi A, Islam AM, Kato S, Wakai S, Kurita H: Mental health, quality of life, and nutritional status of adolescents in Dhaka, Bangladesh: Comparison between an urban slum and a non-slum area. Social Science \& Medicine 2006, 63:1477-1488.

35. Thomas P: Poverty reduction and development in Cambodia: Enabling disabled people to play a role. Disability Knowledge and Research; 2005.

36. Thomas P: Mainstreaming disability in development: India country report. Disability Knowledge and Research 2005.

37. Thomas P: Mainstreaming disability in development: Country-level research- Rwanda country report. Disability Knowledge and Research 2005.

38. VanLeit B, Channa S, Rithy P: Children with disabilities in rural Cambodia: An Examination of functional status and implications for service delivery. Asia Pacific Disability Rehabilitation Journal 2007, 18(2):33-48.

39. Department For International Development: Disability, Poverty and Development London: DFID; 2000, 1-17.

40. Elwan A: Poverty and disability: A survey of the literature. Social Protection Discussion Paper Series Washington DC: The World Bank; 1999.

41. Mitra S: Disability and social safety nets in developing countries. Social Protection Discussion Paper Series Washington DC: The World Bank; 2005, 45.

42. Andreassen R, Skøien R, Øderud T: Preliminary Study: Children with mobility limitations in Southern Africa Norad, SINTEF and Norsk Form; 2006.

43. Baylies C: Disability and the notion of human development: Questions of rights and capabilities. Disability \& Society 2002, 17(7):725-739.

44. Dudzik P, Elwan A, Metts R: Disability policies, statistics, and strategies in Latin America and the Caribbean: A review New York: Inter-American Development Bank (IDB); 2000.

45. Miles S: Engaging with the Disability Rights Movement: The experience of community-based rehabilitation in southern Africa. Disability \& Society 1996, 11(4):501-517.

46. Smith AW: WHO activities for prevention of deafness and hearing impairment in children. Scandinavian Audiology 2001, 30(2):93-100.

47. Gordon D, Parker R, Loughran F, Heslop P: Disabled Children in Britain: A ReAnalysis of the OPCS Disability Surveys London: The Stationery Office; 2000.

48. Blackburn CM, Spencer NJ, Read JM: Prevalence of childhood disability and the characteristics and circumstances of disabled children in the UK: seconary analysis of the Family Resources Survey. BMC Pediatrics 2010, 10(21)

49. Emerson E, Shahtahmasebi S, Lancaster G, Berridge D: Poverty transitions among families supporting a child with intellectual disability. Journal of Intellectual and Developmental Disability 2010, 35(4):224-234.

50. Newacheck PW, Yi Hung Y, Park MJ, Brindis CD, Irwin CE Jr: Disparities in adolescent health and health care: does socioeconomic status matter? Health Services Research 2003, 38(5):1235-1252 
51. Newacheck PW, Halfon N: Prevalence and impact of disabling chronic conditions in childhood. American Journal of Public Health 1998, 88(4):610-617.

52. Bor W, Najmen JM, Andersen J, Morrison J, Williams G: Socioeconomic disadvantage and child morbidity: an Australian longitudinal study. Social Science and Medicine 1993, 36(8):1053-1061.

53. Leonard H, Petterson B, De Klerk N, Zubrick SR, Glasson E, Sanders R, Bower C: Association of sociodemographic characteristics of children with intellectual disability in Western Australia. Social Science \& Medicine 2005, 60(7):1499-1513.

54. Hjern A, Weitoft G, Lindbald F: Social adversity predicts ADHD-medication in school children: a national cohort study. Acta Paediatrica 2010, 99(6):920-924.

55. Berntsson LT, Kohler L: Long-term illness and psychosomatic complaints in children aged 2-17 years in the five Nordic countries. Comparison between 1984 and 1996. European Journal of Public Health 2001, 11(1):35-42.

56. Loeb M, Eide A: Living conditions among people with activity limitations in Malawi: A national representative study Oslo: SINTEF Health Research; 2004.

57. Eide A, Nhiwathiwa S, Muderedzi J, Loeb M: Living conditions among people with activity limitations in Zimbabwe: A representative regional survey Oslo: SINTEF Unimed; 2003.

58. Eide A, Van Rooy G, Loeb M: Living conditions among people with disabilities in Namibia. A national representative study Oslo: SINTEF Unimed; 2003.

\section{Pre-publication history}

The pre-publication history for this paper can be accessed here: http://www.biomedcentral.com/1471-2431/11/119/prepub

doi:10.1186/1471-2431-11-119

Cite this article as: Simkiss et al:. Childhood disability and socioeconomic circumstances in low and middle income countries: systematic review. BMC Pediatrics 2011 11:119.

\section{Submit your next manuscript to BioMed Central and take full advantage of:}

- Convenient online submission

- Thorough peer review

- No space constraints or color figure charges

- Immediate publication on acceptance

- Inclusion in PubMed, CAS, Scopus and Google Scholar

- Research which is freely available for redistribution

Submit your manuscript at www.biomedcentral.com/submit
C BioMed Central 